\title{
Vein Pattern Visualization Through Multiple Mapping Models and Local Parameter Estimation for Forensic Investigation
}

\author{
Hamid Reza Sharifzadeh* \\ Department of Computing \\ Unitec Institute of Technology \\ Auckland, New Zealand \\ e-mail: hsharifzadeh@unitec.ac.nz
}

\author{
Hengyi Zhang, Adams Wai-Kin Kong \\ School of Computer Engineering \\ Nanyang Technological University \\ Singapore \\ e-mail: \{Hengyi, adamskong\}@ntu.edu.sg
}

\begin{abstract}
Forensic investigation methods based on some human traits, including fingerprint, face, and palmprint, have been developed significantly, but some major aspects of particular crimes such as child pornography still lack of notable research efforts. Unlike common forensic identification methods, techniques for identifying criminals in child pornographic images should be developed based on partial non-facial skin observable in the images because criminals always hide their faces. Few methods published recently have shown the potential of vein patterns visualized from color images as a criminal and victim identification tool. However, these methods have two weaknesses: 1) they use single model to visualize vein patterns hidden in color images, which neglects the diversity of skin properties and 2) even though their parameters are determined automatically by an optimization, they do not adapt to fit local image characteristics. To address these weaknesses, this paper proposes an algorithm composed of a bank of mapping models which transform color images to near infrared (NIR) images for visualizing vein patterns and a local parameter estimation scheme for handling different image characteristics in different regions. Imbalanced data regression is also used to systematically construct the model bank. The proposed algorithm is examined and compared with the previous methods on a database of 920 thigh images from 230 subjects. It outperforms the previous methods.
\end{abstract}

Keywords - Vein patterns, skin marks, forensics, biometrics.

\section{INTRODUCTION}

Providing efficient identification technology for the crimes such as child pornography, which has been announced as one of the fastest growing crimes [1], is absolutely amongst the immediate needs of law enforcement agencies around the world. Child pornography is linked to a chain of other serious crimes from human trafficking to under-age sex and sexual abuse. The fight against this crime has currently grabbed the attention of human right activists, media, and nongovernmental organizations around the world [2, 3] and it is officially backed by international organizations [4] and most governments such as UK [5].

Even though DNA, face, fingerprint, and palmprint are amongst the most common biometric traits employed in wellestablished identification systems, these traits are not applicable to child pornographic images, where criminals

*All his work for this paper was done at Nanyang Technological University, Singapore. usually cover their faces and only non-facial skin is partially observable. Lack of such effective identification technology on one side, and the rapid growth of online tools which facilitate production and transmission of child pornographic images on the other side, cause the increase of child victims in sex trafficking and pornography [6, 7]. By developing novel technological tools which help law enforcement agencies identify these criminals, researchers can take a stand against such a growing crime.

Early steps toward this aim have been taken by visualizing vein patterns hidden in color skin images through the opticalbased vein uncovering (OBVU) method [8] and its extension [10] based on Kubelka-Munk theory [11] for modeling the skin color formation. Because vein patterns are usually clear in near infrared (NIR) images, an image mapping method which uses a neural network to map RGB values in color images to NIR intensity values was also proposed [9]. Although these methods give some encouraging results, they have two problems: 1) they use single model to visualize vein patterns hidden in color images, which neglects the diversity of skin properties and 2) their parameters do not adapt to fit local image characteristics, which neglects the local illumination and pose variations. To address these problems, this paper proposes an algorithm composed of a bank of mapping models which transform color images to NIR images for vein visualization and a local parameter estimation scheme for variations of local image characteristics. To systematically construct the mapping models in the model bank and properly deal with the fact that the number of generic skin pixels is always much greater than the number of vein pixels, imbalanced data regression is utilized.

The rest of this paper is organized as follows. Section II presents the related works. Section III explains the construction of the model bank based on imbalanced data regression. Section IV discusses the proposed algorithm. Section V reports the experimental results. Section VI offers conclusive remarks.

\section{LITERATURE REVIEW}

Visualizing vein patterns from color images for criminal and victim identification is a very new research direction. Only limited works on this topic have been published. Tang et al.'s work called the optical-based vein uncovering (OBVU) method was the first attempt [8]. They used Kubelka-Munk (K-M) 
theory to model the optical behavior of the skin and a large number of biophysical parameters from the medical literature for simulating skin color formation, which was considered as a forward model. This forward model also needs spectrum information of the sensor in the camera which takes the image and the illuminant which lights the skin. Tang et al. inputted a range of skin parameters, including the concentration of melanin, the concentration of hemoglobin, and the depth of dermis to the forward model in order to obtain corresponding RGB values. They used these correspondences to train a neural network, which takes RGB values as inputs to predict the skin parameters. Blood in veins have higher concentration of hemoglobin and therefore, vein patterns can be visualized through this method. In their experiments, vein patterns can also be seen in other parameter maps i.e., the concentration of melanin and depth of dermis. Fig. 1 illustrates the OBVU method.

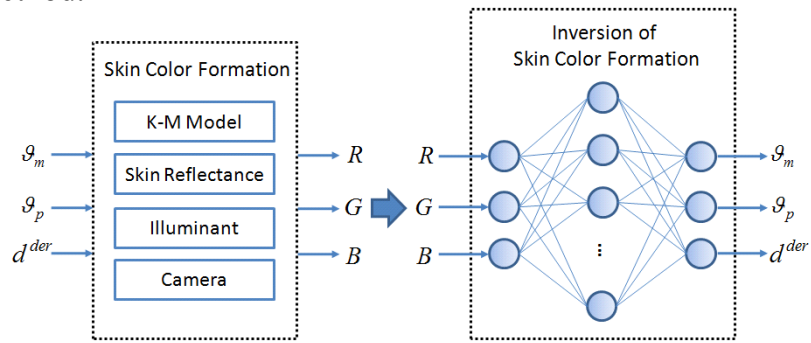

Fig. 1. A schematic diagram of the OBVU method to visualize vein patterns. The left block represents the skin color formation model (the forward model) and the right block represents the inversion of the skin color formation (the backward model) to obtain $\left[\vartheta_{m}, \vartheta_{p}, d^{d e r}\right]$ from $[R, G, B]$, where $\vartheta_{m}, \vartheta_{p}, d^{d e r}$ are respectively the concentration of melanin, hemoglobin, and the depth of dermis

Zhang et al. noted that the OBVU method has two problems: 1) it cannot handle brigthness variation and 2) it was evaluated only on a very small database manually [10]. To resolve these problems, Zhang et al. introduced a parameter $a$ into the trained neural network of the OBVU method. Mathematically, it can be described as

$$
d_{p}=\varphi\left(M_{p} / a\right)
$$

where $M_{p}$ is an RGB value of the pixel $p$ in an input color image, $d_{p}$ is a skin parameter given by the OBVU method, $\varphi$ represents the trained neural network from the OBVU method and $a$ is a free parameter to handle illumination variation. Combining all $d_{p}$ from different pixels forms a vein pattern image denoted as $d$. To optimize the parameter $a$ for different images, the objective function

$$
a^{*}=\underset{a \in A}{\arg \max }\left\{\frac{1}{N} \sum_{i=1}^{N} \underset{(x, y) \in M_{b_{i}}}{\operatorname{var}}\left[d_{b_{i}}(x, y)\right]\right\},
$$

where $a^{*}$ is the optimal $a, A$ is a set of different intensity adjustment ratios, $d_{b_{i}}$ is the $i^{\text {th }} 5 \times 5$ block in $d, N$ is the total number of blocks, $(x, y)$ is the position of a pixel, and $M_{b_{i}}$ is the set of skin pixels in $d_{b_{i}}$ and var represents the operator calculating the local variances. In addition, Zhang et al. proposed a vein extraction method and a three-step vein matching method for vein pattern identification and evaluation. Their method is called improved OBVU method.
Tang et al. took another approach to generate the neural network for vein visualization [9]. They took a pair of synchronized color and NIR images, which are perfectly aligned, to train the neural network. More precisely, the RGB values in the color image were used as inputs and the intensity values in the NIR image were used as target outputs to train the neural network. They noted that the trained neural network is sensitive to the noise in the green channel. Let $W^{i}=$ $\left[\begin{array}{lll}w_{R}^{i} & w_{G}^{i} & w_{B}^{i}\end{array}\right]$ be the weight matrix of the input layer of the trained neural network. The three columns of $W^{i}$ correspond to the input $R, G$, and $B$ values. They added a free parameter $c$ to $W^{i}$ in order to increase its robustness. More precisely, $W^{i}$ is modified as

$$
\widetilde{W}^{i}=\left[\begin{array}{lll}
w_{R}^{i} & w_{G}^{i} \cdot c & w_{B}^{i}
\end{array}\right] .
$$

To optimize the parameter $c$, they used Zhang et al.'s vein extraction method [10], which generates skeletonized vein patterns. The average size of vein segments in the skeletonized image was used as an objective function to determine the optimal adjustment ratio $c^{*}$ :

$$
c^{*}=\arg \max _{c \in C}\left\{\frac{1}{E} \sum_{i=1}^{E} S\left(v_{i}\right)\right\},
$$

where $\boldsymbol{C}$ is a set of different adjustment values, $v_{i}$ is the $i^{\text {th }}$ vein segment in the skeletonized image, $E$ is the total number of vein segments in the image, and $S$ is a function calculating the size of the vein segments. $S$ is defined as:

$$
S\left(v_{i}\right)=\sqrt{\left(r_{\max }-r_{\min }\right)^{2}+\left(c_{\max }-c_{\min }\right)^{2}},
$$

where $r_{\max }\left(r_{\min }\right)$ and $c_{\max }\left(c_{\min }\right)$ are respectively the maximum (minimum) row and column coordinates in the vein segment $v_{i}$. Fig. 2 shows a resultant image and its skeletonized image from this method. In this paper, it is called IR mapping method.

All these vein visualization methods have two common weaknesses. They all are a single model approach, which cannot handle the diversity of skin. Although free parameters are used to enhance their robustness for different lighting conditions, all these parameters are optimized globally and local image and skin characteristics are neglected. This paper proposes a local parameter estimation scheme and a model construction scheme based on imbalanced data regression to overcome these weaknesses.

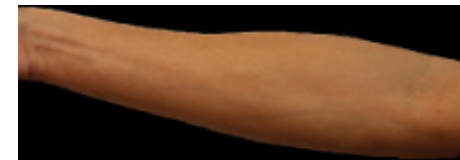

(a)

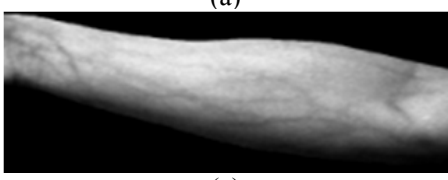

(c)

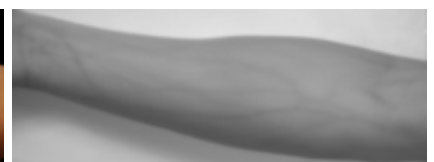

(b)

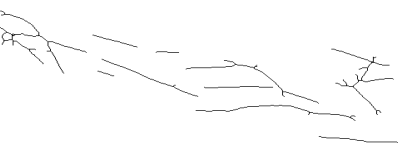

(d)
Fig. 2. A resultant image given by the IR mapping method proposed by Tang et al. [9]. (a) A color forearm image, (b) the corresponding NIR image of (a) for validation, (c) the resultant image given by the IR mapping method and (d) a skeletonized image used in the optimization of Eq. 4 . 


\section{A MODEL BANK GENERATION BASED ON IMBALANCED DATA REGRESSION}

To handle diverse skin, synchronized color and NIR images are used to create a model bank, which contains different models to map input color images to NIR images for vein visualization. It is noted that the neural network in the IR mapping method is directly trained by a pair of synchronized color and NIR images. Their training method is suboptimal because skin images have a lot of generic skin pixels, but limited vein pixels, which cause an imbalanced regression problem. This problem was neglected in the IR mapping method. Furthermore, the IR mapping method does not process the training images i.e., the pair of synchronized color and NIR images. Thus, vein patterns in the training images are not enhanced.

The popular learning methods are reported to be inadequate when encountering the class imbalanced problem [12, 13, 14]. To handle our imbalanced problem, where the majority pixels are from generic skin but vein pixels are relatively rare, we use a data-level solution by combining resampling [15] and boosting [16] approaches. We first boost the vein pixels and resample our bi-class training data. Vein pixels, the minority class, are over-sampled and generic skin pixels, the majority class, are under-sampled by cutting down the images to obtain an optimal class size ratio of 1:1. The block diagram and the corresponding images of these processes are highlighted in Figs. 3 and 4, respectively.

As shown in Fig. 3 and the first row of Fig. 4, we use synchronised color and NIR images taken by a 2-CCD multispectral camera as the raw training data to create a neural network mapping model. The camera simultaneously measures visible and NIR light spectrums through a single lens using two channels. The first channel has a Bayer mosaic color imager that only captures visible light, while the second channel has a monochrome imager for NIR light. The color and NIR images are perfectly registered and synchronised. The input color images are then enhanced through RGB adjustment to emphasize on vein pixels and the corresponding NIR images are also intensified through a filter bank made up of the real parts of 16 Gabor filters [17] with different scales and orientations (the second row of Fig. 4). Then the enhanced synchronised images are resampled to obtain the optimal class ratio of 1:1. (the third row of Fig. 4).

Five mapping models from different thigh image pairs are created by the back propagation method [18] and a three layer feed-forward neural network [19] with 5 hidden neurons. The processed RGB values in the color images and the corresponding intensity values in the NIR images are used respectively as the input and the target output in the training process. The trained models form a model bank for visualizing vein patterns from diverse skin images.

\section{The Proposed Vein Visualization Algorithm}

The proposed vein visualization algorithm first preprocesses the input images to minimize illumination influence, and then selects an optimal model from the model bank. The optimal model is applied to different regions in the preprocessed images to determine regional parameters. These regional parameters are interpolated to obtain local parameters. Finally, the selected optimal model and the local parameters are applied to the preprocessed images simultaneously. The proposed algorithm uses the intensity adjustment scheme given in Eq. 2 and the green channel adjustment scheme given in Eq. 4 for model selection and parameterization. Fig. 5 illustrates the proposed vein visualization algorithm.

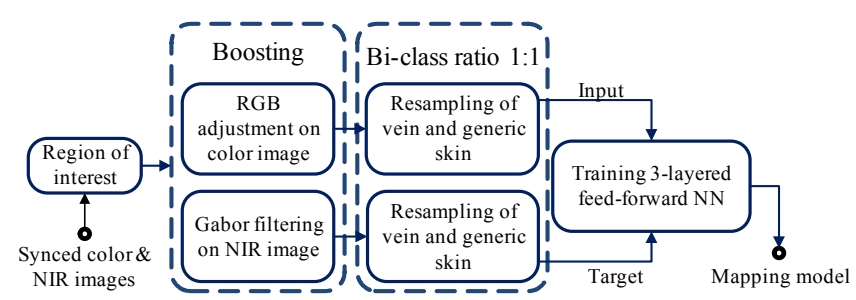

Fig. 3. A block diagram of the NN mapping model generation with boosting and resampling of imbalanced data.
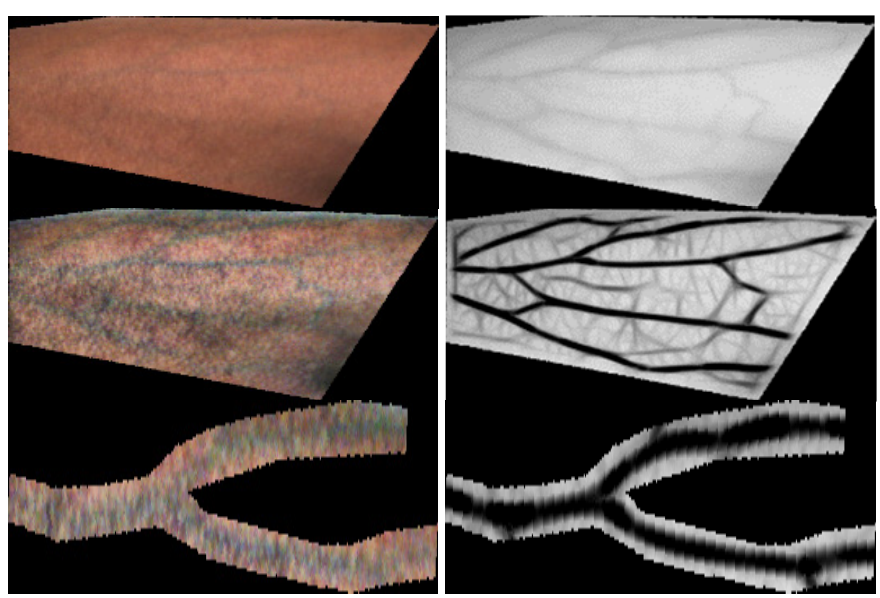

Fig. 4. Illustration of preparing color and NIR images for training NN model. The $1^{\text {st }}-3^{\text {rd }}$ rows respectively show region of interest, enhancement, and resampling by cutting down.

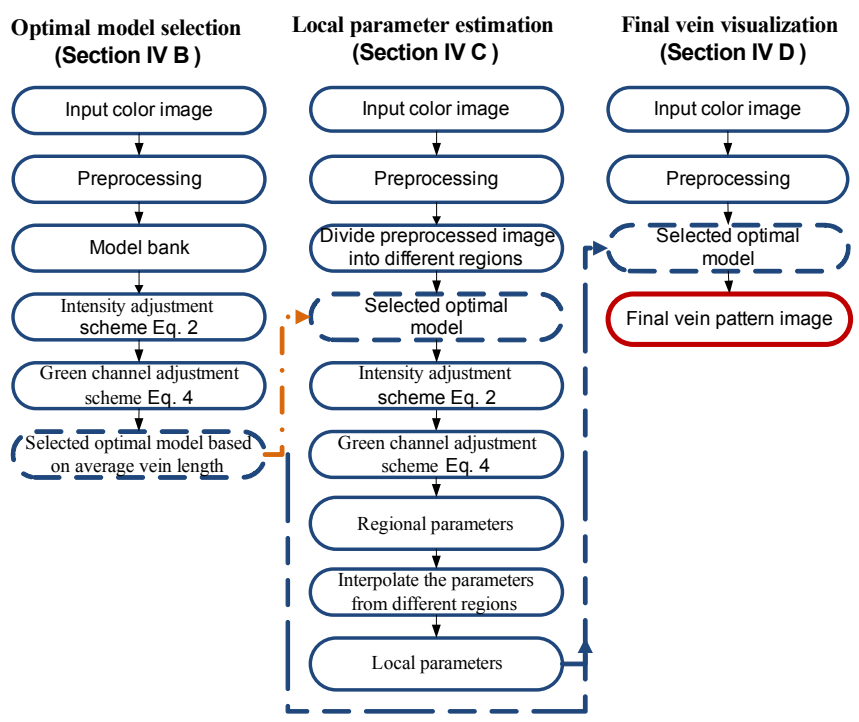

Fig. 5. A block diagram of the proposed vein uncovering algorithm. (a) Optimal model selection, (b) local parameter estimation. 


\section{A. Preprocessing}

To deal with illumination and contrast variations, the color images are first adjusted in the LAB and RGB color spaces before going through other processes. The pre-processing module uses a standard range of light amount (L) in the LAB color space (obtained from the balanced images in our database; i.e. the average amount of $\mathrm{L}$ from images with consistent lighting and no shadows) to adjust the illumination of the input image. Then, the contrast of the input image is adjusted by the adaptive histogram equalisation in the RGB color space.

\section{B. Model Selection}

Let the preprocessed image be $I$ and a trained neural network in the model bank be $\varphi_{k}$. Applying Eq. 2 to $I$, the optimal parameter $a$ for the model $\varphi_{k}$, which is denoted as $a_{k}^{*}$, is obtained. It should be emphasized that each $\varphi_{k}$ produces one $a_{k}^{*}$. Then, the green channel adjustment scheme given in Eq. 4 is applied to $I / a_{k}^{*}$ for the model $\varphi_{k}$ and the optimal parameter $c^{*}$ denoted as $c_{k}^{*}$ for the model $\varphi_{k}$ is obtained. For each model, a pair of parameters $a_{k}^{*}$ and $c_{k}^{*}$ is obtained. The optimal model $\varphi^{*}$ is selected based on average vein length criterion given in Eq. 4. In this selection, each model $\varphi_{k}$ uses their corresponding optimal parameters $a_{k}^{*}$ and $c_{k}^{*}$ to generate skeletonized images for measuring the average vein length. In this model selection, all the parameters $a_{k}$ and $c_{k}$ and model $\varphi_{k}$ are applied to entire input image as with the previous methods.

\section{Local Parameter Estimation}

To estimate the local parameters, the preprocessed images are divided into eight ( 2 by 4 ) non-overlapping regions which are considered as independent images. Let $I_{j}$ where $j \in$ $\{1, \ldots, 8\}$ be one of the regions from the preprocessed image $I$. The intensity adjustment scheme given in Eq. 2 and the green channel adjustment scheme given in Eq. 4 are applied with the optimal model $\varphi^{*}$ to all $I_{j}$. Consequently, eight pairs of parameters $\left\{a_{o j}^{*}, c_{o j}^{*}\right\}$, where $o$ denotes the parameters obtained from the optimal model, are estimated. $a_{o j}^{*}$ and $c_{o j}^{*}$ are called regional parameters. If we apply the same parameter pair to entire $I_{j}$, blocking artifacts will appear. Thus, $\left\{a_{o j}^{*}, c_{o j}^{*}\right\}$ is considered as the parameter pair in the center of $I_{j}$. Other parameter pairs, e.g., around borders of $I_{j}$, are estimated through interpolation. More clearly, through interpolation, different pixels in the preprocessed $I$ have different $a$ and $c$ values. Fig. 6 illustrates the different regions, the centers of the regions with the parameters $\left\{a_{o j}^{*}, c_{o j}^{*}\right\}$ and other parameters obtained through interpolation.

\section{Final Vein Visualization.}

After estimating the local parameters, each pixel $p$ in $I_{j}$ associates with a pair $\left(\tilde{a}_{o p}^{*}, \tilde{c}_{o p}^{*}\right)$, where $\sim$ denotes that they are obtained through interpolation. Finally,

$$
d_{p}=\varphi_{c_{p}}^{*}\left(I_{p} / \tilde{a}_{o p}^{*}\right),
$$

where $d_{p}$ is the predicted NIR intensity value and $\varphi_{c_{p}}^{*}$ is the optimal model $\varphi^{*}$ with the weight matrix $\widetilde{W}^{*}=$ $\left[\begin{array}{lll}w_{R}^{*} & w_{G}^{*} \cdot \tilde{c}_{o p}^{*} & w_{B}^{*}\end{array}\right]$ in the input layer. The original weight matrix in the input layer of $\varphi^{*}$ is $W^{*}=\left[\begin{array}{lll}w_{R}^{*} & w_{G}^{*} & w_{B}^{*}\end{array}\right]$.

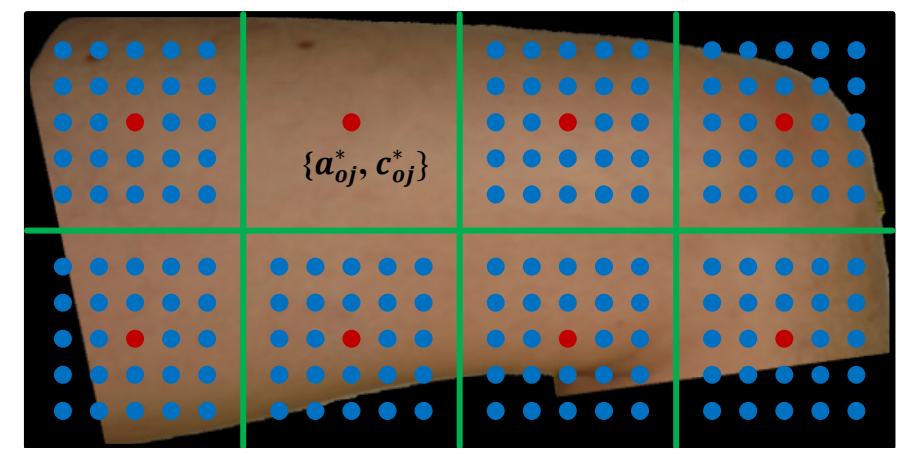

Fig. 6. Illustration of local parameter estimation. The different regions are denoted by green lines. The centers of the regions with the parameters $\left\{a_{o j}^{*}\right.$, $\left.c_{o j}^{*}\right\}$ are denoted by red dots and the other parameters are denoted by blue dots.

Collecting $d_{p}$ from different pixels in the preprocessed image $I$, a vein visualized image is finally produced.

\section{EXPERIMENTAL RESULTS}

The performance of the proposed algorithm is discussed in this section and the results are compared with the improved OBVU method and the IR mapping method $[9,10]$. The threestep matching algorithm described in [10] is used to examine the proposed algorithm. 460 color thigh images and 460 NIR thigh images were collected from 230 different subjects. The images were collected on two separate sessions with an interval of 11 days. In each session, one color and one NIR images were collected from each subject (so totally 920 images from 230 persons). Nikon D70s and Canon 500D were used to capture the color images and JAI camera was used to capture the NIR images. Note that NIR images are used for comparison only. The proposed algorithm does not need the NIR images for vein visualization. Fig. 7 shows some raw images in our database. The skin regions in the NIR and color images were segmented for these experiments.

All the color images were fed into the proposed algorithm and then the extracted vein patterns were used for matching and generating the cumulative matching characteristic (CMC) curves. The 230 visualized images from the first session were matched against another 230 visualized images extracted from the second session. To evaluate the proposed algorithm, the same matching process has been carried out on the visualized images obtained from the previous methods, including the IR mapping method [9] and the improved OBVU method [10] and the results in terms of CMC curves is shown in Fig. 8. Furthermore, matching has been done on two sets of NIR images as well and the corresponding CMC curve is also given in Fig. 8 for comparison. Since the NIR images are usually considered as the ground truth of vein patterns, this result can be used as a reference for indicating the performance of the proposed algorithm. Fig 9 shows resultant images from different methods for visual comparison. The vein pattern given by the proposed algorithm is the clearest and it handles uneven illumination much better than other methods.

Table 1 summarizes the identification accuracy from matching two sets of NIR images with NIR images and two sets of color images with color images which are visualized by the proposed algorithm, the IR mapping method, and the 
improved OBVU method. The term "rank-10\%" identification accuracy refers to the percentage of input vein patterns (from the first session) whose corresponding vein patterns (from the second session) can be found within the top $10 \%$ of the total vein patterns in the gallery set. The identification accuracy in Table 1 and the CMC curves in Fig. 8 show that the proposed algorithm outperforms the previous vein visualization methods.

TABLE I. IDENTIFICATION ACCURACY FROM DIFFERENT METHODS.

\begin{tabular}{|c|c|c|}
\hline Method/Type & Rank-One & Rank-10\% \\
\hline NIR Images & $57 \%$ & $71 \%$ \\
\hline IR mapping & $29 \%$ & $43 \%$ \\
\hline Improved OBVU & $33 \%$ & $50 \%$ \\
\hline Proposed algorithm & $46 \%$ & $61 \%$ \\
\hline
\end{tabular}

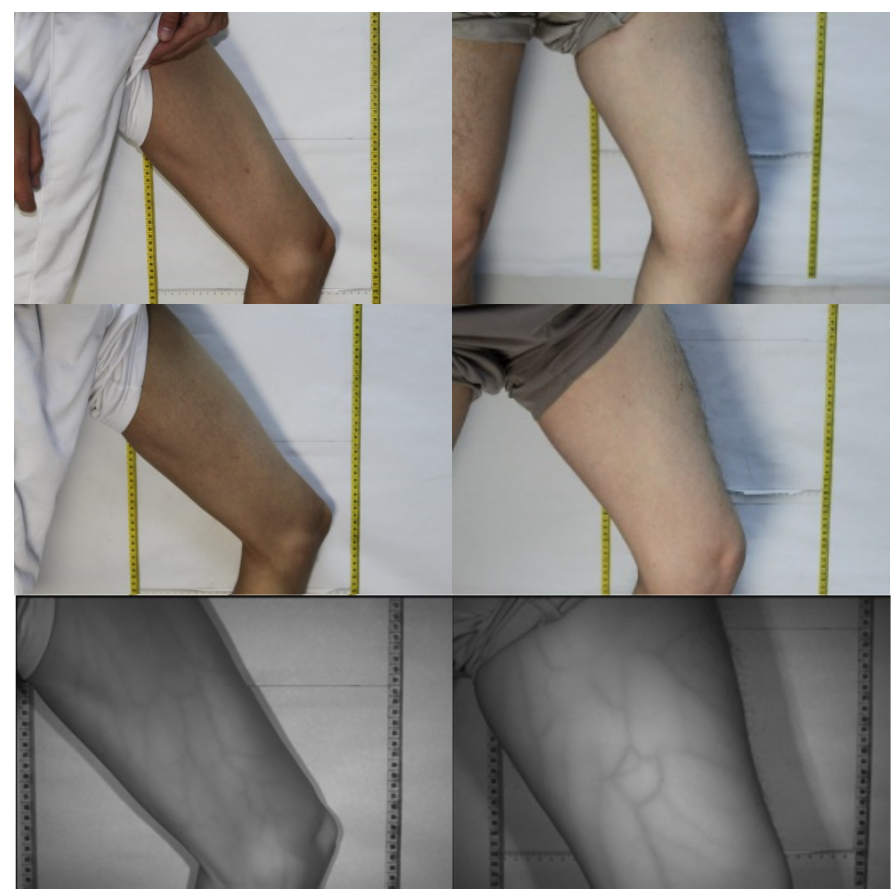

Fig. 7. Two sets of raw images in our database. Each column shows images from the same leg. Color images in the first and the second rows were collected from the first and the second sessions, respectively. The last row is the corresponding NIR images.

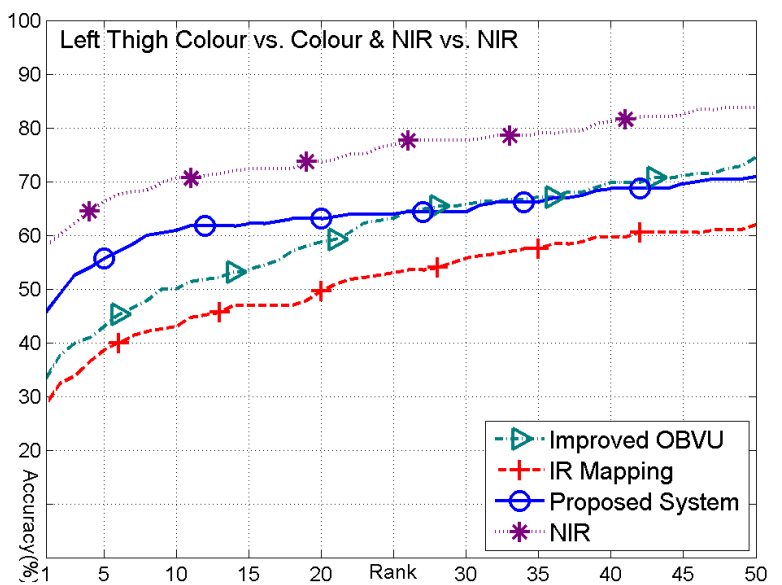

Fig. 8. Cumulative matching characteristic curves from the same type of images (color vs. color and NIR vs. NIR) with different visualization methods.

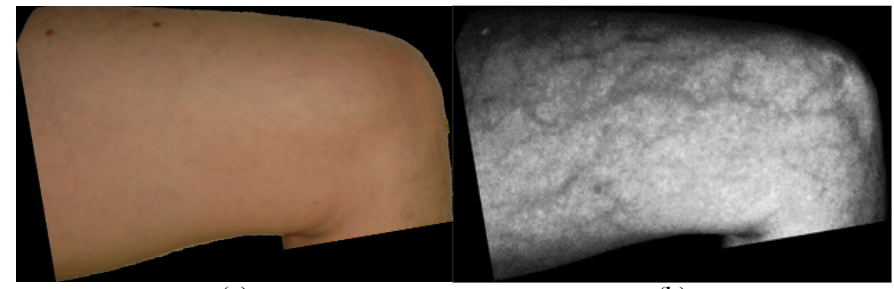

(a) (b)

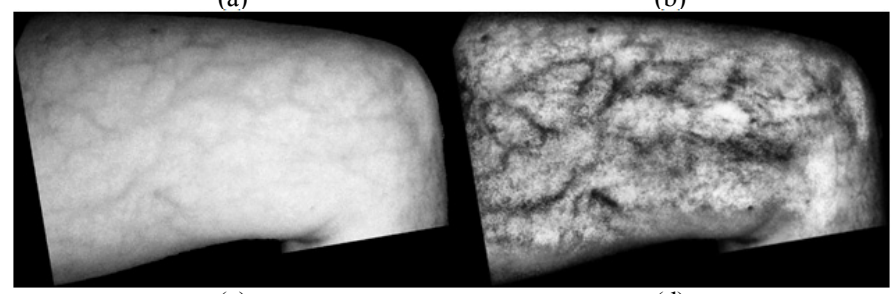

(c)

(d)

Fig. 9. A visual comparison of the proposed algorithm and the previous methods: (a) is a color image of a thigh and (b)-(d) are resultant images from (b) the improved OBVU [10], (c) the IR mapping [9] and (d) the proposed algorithm, respectively.

\section{CONCLUSION}

To overcome the limits in the previous vein visualization methods for forensic investigation, this paper proposes an algorithm composed of a bank of mapping models, which transform color images to NIR images for visualizing vein patterns and a local parameter estimation scheme for handling different image characteristics in different regions. Imbalanced data regression is also used to eliminate the weaknesses of the model construction scheme employed by the IR mapping method. The proposed algorithm first selects an optimal model from the model bank and then estimates local parameters for each pixel in the input image to enhance the visualization performance. It has been evaluated on a thigh image database with 460 color images and 460 NIR images from 230 subjects. The experimental results indicate that the proposed algorithm outperforms the previous vein visualization methods.

\section{ACKNOWLEDGMENT}

The authors would like to thank the Ministry of Education, Singapore for partial support of this research through Academic Research Fund Tier 2, MOE2012-T2-1-024. We also would like to thank the U.S. Department of Justice, the U.S. Immigration and Customs Enforcement, the Singapore Prison Service, and the Singapore Police Force for providing legal and forensics information.

\section{REFERENCES}

[1] M. Motivans and T. Kyckelhahn. Federal prosecution of child sex exploitation offenders 2006. Technical report, US Department of Justice, Office of Justice Programs, Bureau of Justice Statistics, 2007.

[2] The cnn freedom project. http:// thecnnfreedomproject.blogs.cnn.com/. [online; accessed October 2013].

[3] Dc stop modern slavery community. http://www.stopmodernslavery.org/. [online; accessed October 2013]

[4] International justice mission. http://www.ijm.org/node/187/. [online; accessed October 2013]. 
[5] Uk backs project to end modern day slavery for girls and women. https://www.gov.uk/government/news/work-in-freedom/. [online; accessed October 2013].

[6] S. Chawla. Global report on trafficking in persons. Technical report, United Nations Office on Drugs and Crime, 2012.

[7] N. Pereda, G. Guilera, M. Forns, and J. Gomez-Benito. The international epidemiology of child sexual abuse: a continuation of finkelhor (1994). Child Abuse \& Neglect, 33(6):331-342, 2009.

[8] C. Tang, A. Kong, and N. Craft. Uncovering vein patterns from color skin images for forensics analysis. In IEEE Computer Vision and Pattern Recognition, pages 665-672, 2011.

[9] C. Tang, A. Kong, and N. Craft. Visualizing vein patterns from color skin images based on image mapping for forensics analysis. In International Conference on Pattern Recognition, pages 2387-2390, 2012.

[10] H. Zhang, C. Tang, A. Kong, and N. Craft. Matching vein patterns from color images for forensic investigation. In IEEE International Conference on Biometrics: Theory, Applications and Systems, pages 77-84, 2012.

[11] P. Kubelka. New contribution to the optics of intensely light-scattering materials. Journal of the Optical Society of America, 38(5):448-457, 1948.

[12] G. E. Batista, R. C. Prati, and M. C. Monard. A study of the behavior of several methods for balancing machine learning training data. ACM SIGKDD Explorations Newsletter Special Issue on Learning from Imbalanced Datasets, 6(1):20-29, 2004.

[13] N. Japkowicz and S. Stephen. The class imbalance problem: a systematic study. Journal of Intelligent Data Analysis, 6(5):429-449, 2002.

[14] Y. Sun, A. K. C. Wong, and M. S. Kamel. Classification of imbalanced data: A review. International Journal of Pattern Recognition and Artificial Intelligence, 23(4):687-719, 2009.

[15] N. V. Chawla, K. Bowyer, L. Hall, and W. P. Kegelmeyer. Smote: Synthetic minority over-sampling technique. Journal of Artificial Intelligence Research, 16:321-357, 2002.

[16] Z. H. Zhou and X. Y. Liu. Training cost-sensitive neural networks with methods addressing the class imbalance problem. IEEE Transactions on Knowledge and Data Engineering, 18(1):63-77, 2006.

[17] A. Kong. An evaluation of gabor orientation as a feature for face recognition. In International Conference on Pattern Recognition, pages $1-4,2008$.

[18] J. Hertz, A. Krogh, and R. G. Palmer. Introduction to the Theory of Neural Computation. Addison Wesley, 1991.

[19] R. Anand, K. G. Mehrotra, C. K. Mohan, and S. Ranka. An improved algorithm for neural network classification of imbalanced training sets. IEEE Transactions on Neural Networks, 4(6):962-969, 1993. 